\title{
Os Indicadores de Desempenho Contratualizados com as USF: Um ponto da situação no actual momento da Reforma
}

Miguel Melo,* Jaime Correia de Sousa**

\section{RESUMO}

Algum tempo passado sobre a introdução dos Indicadores de Desempenho no processo de contratualização e avaliação de desempenho das USF, importa reflectir sobre a qualidade e seu o impacto na prestação de cuidados na Medicina Geral e Familiar (MGF), com a finalidade de contribuir para a melhoria da qualidade da avaliação de desempenho.

Ao restringir a actividade avaliada pelos Indicadores a uma pequena área da prática clínica e ao contratualizar metas com valores muito elevados, pode conduzir ao afunilamento e à focalização da actividade clínica, com eventuais consequências negativas para os utentes. Uma das consequências possíveis é a prática de uma Medicina Baseada em Indicadores em vez de uma Medicina Centrada no Doente.

Por outro lado, a fraca evidência científica de alguns Indicadores, a utilidade muito discutível de outros, bem como a dificuldade em medir ganhos em saúde podem originar excessos da Medicina, nomeadamente medicalização, consumismo e iatrogenia.

São feitas, neste artigo, algumas sugestões sobre alguns aspectos a melhorar nos Indicadores. Outros aspectos relacionados, tal como o Sistema de Informação e a Contratualização terão de evoluir paralelamente.

O Ideal seria que o pagamento por desempenho fosse baseado na medição de Indicadores que representem ganhos em Saúde para as pessoas, respeitando igualmente os princípios e a prática da Medicina Geral e Familiar.

Palavras-chave: Reembolso de Incentivo; Indicadores de Qualidade em Assistência à Saúde; Garantia da Qualidade dos Cuidados de Saúde.

\section{INTRODUÇÃO}

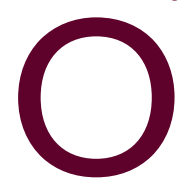

desenvolvimento da Medicina Geral e Familiar (MGF) Portuguesa ao longo dos últimos vinte anos tem sido acompanhado pelo debate em torno da optimização dos modelos organizacionais e, consequentemente, dos sistemas retributivos com eles relacionados que permitam uma retribuição mais justa dos profissionais, ao mes-

*Médico de Família na USF de Fânzeres, ARS Norte

**Médico de Família na USF Horizonte, ULS Matosinhos, Professor Auxiliar Convidado da Escola de Ciências da Saúde da Universidade do Minho. mo tempo que se associam a uma melhoria da qualidade da prática clínica.

Os líderes da Medicina Geral e Familiar Portuguesa foram observando a evolução dos sistemas de pagamento nos diferentes Países e sistemas de saúde e lançaram o debate sobre as formas mais adequadas e a adaptação possível à legislação Nacional. Essas reflexões, sistematizadas em alguns artigos publicado ${ }^{1-7} l e-$ varam à produção de vários documentos orientadores protagonizados pela Associação Portuguesa dos Médicos de Clínica Geral (APMCG) que foram sendo entregues aos sucessivos responsáveis do Ministério da 
Saúde até terem conseguido influenciar a produção legislativa. ${ }^{8-10}$

Um dos princípios mais importantes defendidos pela APMCG no documento de 1990 «Um futuro para a Medicina de Família em Portugal», ${ }^{8}$ era o de que os contratos deveriam ser feitos «com médicos que se proponham trabalhar em grupo e numa perspectiva de trabalho multiprofissional» e recomendava já a existência de vários componentes da remuneração.

O diploma legislativo que introduziu pela primeira vez um novo sistema retributivo o Regime Remuneratório Experimental (RRE) ${ }^{11}$ consagrou o princípio há tanto tempo defendido pela APMCG, de descriminação positiva associada a quantidade e qualidade de trabalho diferente, conceito este mais tarde reforçado no Decreto-lei que criou as Unidades de Saúde Familiar ${ }^{12}$ e no Despacho Normativo de $2006^{13}$ que aprovou o regulamento para lançamento e implementação das USF.

No Documento da APMCG de 1998, a Declaração da Madeira, ${ }^{9}$ o princípio da avaliação individual de desempenho é claramente defendido numa «perspectiva voluntária e periódica, sendo necessário identificar em reuniões de trabalho, por métodos de consenso, quais os parâmetros e indicadores a utilizar, construir uma matriz de referência para este processo de avaliação de desempenho e organizar um dispositivo de apoio logístico à sua operacionalização, registo e certificação apoiado em protocolos de orientação clínica, baseados em critérios de evidência científica».

Os indicadores de desempenho surgem pela primeira vez consagrados em diploma legislativo com a designação de indicadores de monitorização para a contratualização no Despacho Normativo 9 de 2006. ${ }^{13}$

Os elementos da Missão para os Cuidados de Saúde Primários (MCSP) desenvolveram um conjunto de indicadores a utilizar no acompanhamento e contratualização com as USF que deram origem ao documento «Indicadores de Desempenho para as Unidades de Saúde Familiar». ${ }^{14}$ Segundo é aí mencionado, terá havido consulta pública através do sítio da Internet da MCSP, mas esteve-se muito longe do processo participativo assente nos próprios profissionais e nas USF defendido nos documentos da APMCG.

Algum tempo passado sobre a introdução e utilização sistemática dos referidos indicadores no processo de contratualização e avaliação de desempenho das
USF, importa reflectir sobre o modelo conceptual que suportou a introdução de um novo sistema retributivo nos cuidados de saúde primários, o pagamento ligado à dimensão da lista de utentes e ao cumprimento de um conjunto de tarefas contratuais, e sobre a qualidade e o impacto dos Indicadores de desempenho na prestação de cuidados na MGF, com a finalidade de contribuir para a melhoria da qualidade da avaliação de desempenho.

\section{Pagamento por desempenho (PpD)}

O pagamento por desempenho $(\mathrm{PpD})$, do termo inglês Pay for Performance (P4P) é o modelo de retribuição dos profissionais de saúde em que estes auferem uma retribuição variável em função do atingimento de determinadas metas pré-estabelecidas para a prestação de cuidados de saúde..$^{15}$

Em muitos sistemas de saúde este método de remuneração correspondeu a uma evolução do modelo de pagamento ao acto. $\mathrm{OPDD}$ pode coexistir com outras formas de retribuição dos profissionais, nomeadamente com pagamento ligado à capitação, algumas formas de pagamento ao acto e a componentes de vencimento fixo. ${ }^{16}$

A redacção da Portaria n. ${ }^{\circ}$ 301/2008 de 18 de Abril estipula que «a contratualização de metas de desempenho com as USF deve conduzir, por si só, a esforços tangíveis na melhoria dos níveis da acessibilidade, dos desempenhos assistencial e económico e da satisfação dos utentes, bem como distinguir as USF que atinjam as metas contratualizadas com a atribuição de recompensas, suportadas pelos ganhos de eficiência previamente incorporados no sistema de saúde». ${ }^{17}$

\section{Pressupostos do PpD}

Os defensores do sistema PpD sustentam este modelo com os pressupostos de que o sistema aumentaria a motivação dos profissionais e traria um incremento das boas práticas com ganhos em saúde. Na realidade, o sistema foi inicialmente desenvolvido com o objectivo de melhoria de qualidade e pressupõe um regime de estímulos ligado a ganhos e incentivos pelo cumprimento de certas metas dentro de um determinada margem de variação aceitável. ${ }^{18}$

A American Academy of Family Physicians (AAFP) reconhece a necessidade de se rever o sistema de pagamento dos médicos, incluindo o pagamento por desempenho reconhecendo contudo que existem muitos 
desafios organizacionais, técnicos, legais e éticos para o desenvolvimento e implementação deste sistema. ${ }^{19}$ Consideram que este tipo de programas tem o potencial de aumentar o uso das tecnologias de informação, de normas de orientação clínica baseadas na evidência, boas práticas clínicas e organizativas e o acesso a cuidados atempados e apropriados por parte dos médicos de família.

A American Academy of Family Physicians (AAFP) apoia o PpD que adira aos seguintes princípios: ${ }^{19} 1$ ) Enfoque na melhoria da qualidade de cuidados. 2) Apoie a relação médico-doente. 3) Utilize medidas de desempenho apoiadas em normas de orientação clínica baseadas na evidência. 4) Envolva médicos de família com prática clínica no seu desenvolvimento. 5) Utilize dados credíveis, exactos e cientificamente válidos. 6) Proporcione incentivos positivos aos médicos e 7) Possibilite a participação voluntária. A AAFP também reconhece que há tanto vantagens como desvantagens neste sistema na forma como são desenvolvidos e implementados actualmente.

\section{Dúvidas e problemas com o PpD}

Apesar das diversas manifestações políticas de apoio ao desenvolvimento do $\mathrm{PpD}$, existem na literatura diversos estudos que põem em causa as vantagens do sistema de $\mathrm{PpD}^{20,21}$ ou levantam questões éticas pertinentes. ${ }^{22,23}$

Numa revisão sobre o tema, Gérvas menciona efeitos adversos do PpD, entre os quais o perigo do aumento das cascatas diagnósticas e terapêuticas. O pagamento por desempenho apresenta graves problemas éticos, entre eles o risco de que o trabalho clínico se passe a centrar no seu cumprimento e que os doentes em cujo seguimento os indicadores se cumpririam com dificuldade sejam afastados, podendo com isso modificar-se a relação médico-doente por o acto clínico se centrar no cumprimento dos indicadores e não nas necessidades do paciente. ${ }^{24}$

Alguns autores referem que o PpD produz poucos ganhos em qualidade para o investimento feito e irão apenas recompensar os que já tinham um elevado nível de desempenho; outros mencionam que não é possível demonstrar a efectividade do sistema. ${ }^{25}$ Mas, em todo o caso, nos estudos revistos parece haver algum efeito positivo do sistema PpD. ${ }^{26}$

\section{INDICADORES DE DESEMPENHO CONTRATUALIZADOS (IDC) COM AS USF}

Em relação aos Indicadores de Desempenho Contratualizados (IDC) no nosso País, ${ }^{27}$ alguns aspectos merecem reflexão:

\section{1. Área restrita}

Ao restringir a área abrangida pelos Indicadores de desempenho contratualizados (IDC) a uma pequena área da nossa actividade existe um claro perigo de afunilamento da actividade médica. Quer o médico de família (MF) quer a Unidade de Saúde Familiar (USF) podem tender a privilegiar as tarefas contratualizadas em detrimento de outras, uma vez que a sua remuneração está dependente dos resultados obtidos. A área abrangida pelos IDC Programas de Vigilância em Saúde Infantil (SI) até aos 2 anos, de Planeamento Familiar (PF), de Saúde Materna (SM), de Hipertensão Arterial (HTA), de Diabetes (DM) e de Rastreio Cancro da Mama e do Colo do útero é manifestamente pouco representativa da globalidade de cuidados da MGF e de uma USF.

De forma a minimizar o afunilamento da actividade médica, deveria existir em Portugal um alargamento dos IDC, tal como em Inglaterra, onde o quadro de Incentivos negociados (QOF: Quality and Outcomes Framework $)^{28}$ em 2009/2010 abrange um painel de cerca de 150 Indicadores. Estes Indicadores abarcam áreas tão distintas como Indicadores clínicos (incluindo Prevenção Secundária e Terciária), dez doenças crónicas (AVC, Asma, DPOC, etc.), internamentos, aspectos qualitativos da prescrição, aspectos organizacionais, actividades educacionais, etc.

\section{Medições de resultados em saúde por aproximação}

O ideal será que os Indicadores meçam resultados clínicos, com interesse para os doentes (POEM) e que representem inequivocamente ganhos em saúde. ${ }^{29}$ No momento actual apenas é possível medir dados do processo (número de consultas efectuadas, número de medições de Tensão Arterial, número de pedidos de HgAlc, etc.) e alguns resultados intermédios (ex: $\mathrm{HgAl}<8 \%$, Tensão Arterial (TA) < 140/90 mmHg). Estes Indicadores não demonstraram ainda a sua efectividade sendo fundamental a sua avaliação em termos de «ganhos em saúde» e não em dados do processo ou intermédios. Esta questão é fundamental para os utentes e para os 
profissionais bem como para a avaliação do sucesso da Reforma dos Cuidados Primários em curso. ${ }^{30}$

\section{Fraca evidência científica das recomendações que su- portam a maioria dos Indicadores}

Como princípio geral os Indicadores escolhidos deveriam ser, sempre que possível, baseados nas melhores evidências de acordo com o paradigma da Medicina Baseada na Evidencia, tentando maximizar ganhos em saúde. O Rastreio do Cancro do Colo do Útero, o Diagnóstico Precoce (RN), follow up de HTA e DM, o Plano Nacional de Vacinação, são alguns dos exemplos de IDC baseados em boas provas científicas. É de estranhar que não se inclua o Rastreio do Cancro Colo Rectal, a Vacina anti Gripal (VAG), conselhos sobre Tabagismo e até follow up de outras patologias importantes (como é o caso da doença cerebrovascular em Portugal) que têm provas dadas e, aparentemente, de simples operacionalização nos Sistemas de Informação. No futuro será de equacionar a introdução destes IDC. Outro aspecto importante é o de não valorizar apenas a actividade de prevenção primária (quase sempre privilegiada) e de privilegiar igualmente aspectos de medicina curativa (incluindo a prevenção terciária). ${ }^{30}$ Alguns dos IDC são, como reconhece a própria MCSP, ${ }^{14}$ baseados nas diferentes Orientações Técnicas produzidas pela Direção Geral de Saúde (DGS), como é o caso dos Indicadores que medem a Periodicidade das Consultas (SI; SM; PF, HTA; DM) bem como a periodicidade do pedido de alguns Meios Complementares de Diagnóstico e Terapêutica (MCDT) (ex.: HgAlc trimestral, ECG anual na DM, etc.). Estas Normas aparentemente são baseadas em consensos e não apresentam as evidências que garantam a sua utilidade.

Um bom exemplo desta questão é espelhado nas recomendações sobre periodicidade das Consultas de HTA. As recomendações não são concordantes existindo recomendações para um follow up semestral, quadrimestral e até trimestral o que terá consequências na organização da prática clínica muito diferentes. A título de exemplo, numa lista de 1880 utentes, com uma prevalência de HTA de $40 \%$ nos utentes com > 18 anos é de esperar cerca de 562 utentes com hipertensão. O número de horas a disponibilizar por semana para a consulta de HTA para estes 562 hipertensos varia de 5 horas/semana (de acordo com as recomendações In- glesas, ${ }^{28}$ uma consulta de 9-9 meses), a 8 horas/ semana (MCSP: uma consulta semestral) ${ }^{31}$ a 12 a 16 horas/ / semana (de acordo com DGS que sugere 3 a 4 consultas/ano). ${ }^{32}$ Esta situação é em tudo idêntica à periodicidade do pedido da HgAlc na vigilância dos utentes com diabetes, com recomendações que variam entre um pedido anual (QOF), semestral, quadrimestral (MCSP) e trimestral.

Verifica-se nos IDC utilizados em Portugal, em comparação com o caso Inglês, uma tendência a fazer mais consultas de HTA e pedir MCDT com mais frequência. Nestas áreas, em que não existem evidências que sustentem estas periodicidades, toda a cautela é necessária, de acordo com o princípio de primum non nocere. Estes aspectos podem fazer toda a diferença: é a diferença entre a utilização dos Serviços com racionalidade, evitando os excessos (Prevenção Quaternária), ou o consumismo, a medicalização, a iatrogenia e o aumento de custos. ${ }^{29,33}$

Existe um grupo de IDC cuja fundamentação, inteligibilidade e utilidade é muito discutível e difícil de entender, para além dos aspectos éticos. Estão neste grupo os IDC que medem as Consultas efectuadas pelo próprio médico (o conceito subjacente da Intersubstituição é muito discutível. E porquê valores de $80 \%$ ?), visitação domiciliária de todos os Recém Nascidos e o registo do IMC das crianças entre os 12-23 meses, independentemente de serem utilizadores ou não da USF. E o que dizer da vacinação antitetânica, em massa, dos utentes com hipertensão? Em relação a custos em Medicamentos e MCDT por Utilizador existem ainda poucas certezas e muitas dúvidas pelo que a utilização destes Indicadores deve ser aperfeiçoada e ajustada, não apenas pelos aspectos economicistas (curvas de percentis dos custos), mas sim por patologia, pela pirâmide etária, pelo nível socioeconómico da população, etc. ${ }^{29,34}$

Em relação aos aspectos éticos é de referir que a questão da exclusão de doentes, nos denominadores dos IDC está ainda por clarificar, podendo originar exclusões dos doentes «piores». Por outro lado, a inclusão de todos os doentes (vigiados ou não na USF) no denominador de alguns IDC parece ser abusiva e susceptível de originar situações bastante discutíveis, como seja a «convocação» de utentes sem compromisso de vigilância com a USF. Importa debater sobre a responsabilidade dos utentes e da USF na adesão dos utentes aos planos de saúde propostos pelos profissionais. ${ }^{35,36}$ 


\section{Os valores das Metas contratualizados para os IDC}

Um aspecto muito importante é o valor das metas. É de esperar que metas muito elevadas, quase utópicas, condicionem a concentração de esforços (focalização) de forma a obter resultados/ incentivos.

Esta situação origina a que se questione sobre o modo como são definidas metas. Como se definem? Os profissionais da USF foram envolvidos? Foram consideradas eventuais consequências perversas para os doentes, decorrentes da focalização e afunilamento? Será que ao exigir que as USF apresentem um valor de $70 \%$ das mulheres com resultados de mamografia, não se estará a confundir as metas do rastreio tipo case finding com as exigências do rastreio sistemático? Não será irrealista exigir que $95 \%$ dos dos utentes com hipertensão tenham 2 registos anuais de Tensão Arterial? Não seria melhor apostar em metas pragmáticas, realistas (afinal lidamos com pessoas, falíveis,...) do que apostar em valores teóricos só imagináveis por quem está longe da prática clínica? No caso Inglês ${ }^{28}$ foram adoptados valores pragmáticos e, no caso da HTA e DM, as metas contratualizadas situam-se entre os 40 e os 80 \% de medição de TA nos últimos 9 meses e medição de HgAlc nos últimos 15 meses!

É muito importante estudar os efeitos do afunilamento e focalização da actividade clínica, provocados pela utilização destes Indicadores e das suas metas, e avaliar o que se deixa de fazer por se fazer o que nos exigem. Este aspecto é tanto mais importante quanto maior a tendência, visível, para um crescimento anual das metas negociadas (apesar do efeito de tecto conhecido). ${ }^{21}$

\section{Medicina centrada nos indicadores}

A implementação dos Indicadores nas USF pode afectar a organização da USF bem como a própria prática de MGF.

A própria ACSS, no documento da Metodologia de contratualização de $2010,{ }^{37}$ adverte que «as metas devem ser exigentes mas viáveis, baseadas nas melhores práticas, (...) que tragam melhores resultados em saúde, sem colocar em causa a implementação e o desenvolvimento organizacional de Equipa».

De acordo com um estudo, ${ }^{38}$ que pretendia avaliar as consequências dos IDC na prática clínica, para se atingir o cumprimento das metas contratualizadas é neces- sário um dispêndio de tempo não exequível pelo MF na prática clínica habitual. Recomenda que se privilegiem as actividades/ indicadores que conduzam a um maior ganho em saúde (ex:tabagismo, Vacina Gripe, uso de IECA na Doença Isquémica Coronária, etc.). De facto parece existir uma dissociação entre os Incentivos e o provável impacto na Saúde, levando a que alguns autores concluam que na maioria das vezes o que se paga é o trabalho e não o potencial benefício para o doente. ${ }^{18,30}$

Por outro lado, a impossibilidade de monitorizar o desempenho da USF, o mau Sistema de Informação e a criação, em algumas USF, de um outro sistema de informação (com o objectivo de automonitorização, mas que obriga a um duplo registo) agravam, e muito, a focalização e o afunilamento da actividade de saúde/USF.

Uma das consequências possíveis é aquilo a que poderemos chamar de Medicina Baseada em Indicadores em contraponto à Medicina Centrada no Doente (um dos pilares fundamentais da nossa prática).$^{35}$ No nosso conceito a Medicina Baseada em Indicadores tem como centro da sua preocupação os números/metas (mais do que as pessoas), tem uma preocupação mais restrita/afunilada (em vez da globalidade, característica da USF), está mais preocupada, para atingir as metas, em convocar pessoas ou até excluir os não cumpridores dos denominadores (em vez de fomentar a autonomia e responsabilização dos doentes), originando questões éticas e conflitos de interesses. ${ }^{35}$

As eventuais repercussões negativas dos IDC na prática de Medicina Geral levou já com que algumas eminentes figuram da nossa área, como Barbara Starfield, recomendem que "é a Medicina Familiar que deve condicionar a Reforma e não o contrário». ${ }^{39}$

\section{O que importa melhorar e desenvolver nos indicadores}

De acordo com o atrás discutido, sugeririmos alguns aspectos a melhorar nos Indicadores: ${ }^{30,34,35,38}$

- Revisão anual dos IDC de forma a aferir a sua validade

- Construção dos Indicadores de acordo com as melhores evidências e de acordo com provas dadas para os doentes (POEM)

- Os IDC devem mudar periodicamente de forma a evitar o afunilamento

- Deve aumentar o número de IDC, de forma alargar 
o seu leque e avaliar a qualidade Global da MGF/ /USF, minimizando assim quer o afunilamento quer a focalização.

- Os IDC devem ser escolhidos entre aqueles que têm maior impacto (ganhos em saúde, custo efectividade, etc.)

- Envolvimento dos profissionais das USF na construção dos Indicadores.

- Rever a metodologia de construção das metas dos IDC, evitando valores muito elevados que podem levar a focalização da prática clínica.

\section{CONCLUSÕES}

A conjugação do alargamento do número de IDC, o alargamento das áreas da prática clínica avaliadas pelos IDC e a redução da fasquia da meta para valores realistas, são aspectos fundamentais para preservar o perfil de actuação de uma Medicina Geral de qualidade. Uma vez que não existem provas convicentes de Ganhos em Saúde com o PpD, importa evitar situações perversas com os Indicadores que podem acarretar consequências menos positivas para os utentes. ${ }^{30,34,35}$

Para além dos Indicadores existem outros aspectos relacionados que urge melhorar tal como o Sistema de Informação, a Contratualização e a avaliação do impacto do PpD para as USF / Cuidados de Saúde Primários nomeadamente o seu custo efectividade, alterações organizacionais da USF e eventuais danos colaterais para os utentes.

O sistema de PpD em Portugal está na sua infância e terá que ser muito aperfeiçoado, á medida que se vai ganhando experiência com a contratualização e se possam extrair do sistema de informação dados fiáveis e credíveis.

Os sistemas de PpD atrás mencionados, tal como o que está consignado na legislação Portuguesa, preconizam sempre o estabelecimento de regimes de estímulos positivos, sem penalização, que premeiem os grupos de profissionais que atingem determinados patamares de desempenho.

\section{REFERÊNCIAS BIBLIOGRÁFICAS}

1. Tavares J F. Os primeiros dez anos da Associação Portuguesa dos Médicos de clínica geral. Lisboa : Departamento Editorial da APMCG, 1997.

2. Sousa JC, Campos L, Miranda M, Lima MAR, Costa MG, Ornelas MM, Galaghar M. Evolução do conceito de centro de saúde no âmbito do novo estatuto do S.N.S. e a criação do nível local de administração de saúde. Acta Med Port 1994; 7(6):385-390.

3. Sakellarides $C$. De Alma-Ata a Harry Potter: um testemunho pessoal. Rev Port S Pública 2001; Volume Temático 2:101-8.

4. Sousa JC, Melo M, Sardinha AM, Perez y Sanchez J, Ribas MJ. Os cuidados de saúde primários e a medicina geral e familiar em Portugal. Rev Port S Pública 2001; Volume Temático 2: 63-74.

5. Branco AG, Ramos V. Cuidados de Saúde Primários em Portugal. Rev Port S Pública 2001; Volume Temático 2: 5-12.

6. Pisco L. Êxitos e Insucessos da Medicina Geral e Familiar Portuguesa ou o que conseguimos em 20 anos e o que falta conseguir. In: Alves MV, Ramos V, editores. Da Memória. Lisboa: MVA Invent / Pfizer/ APMCG, 2003. p. 15-23.

7. Biscaia AR. A reforma dos cuidados de saúde primários e a reforma do pensamento. Rev Port Clin Geral 2006;22:67-79

8. Associação Portuguesa dos Médicos De Clínica Geral, direcção Nacional. Um futuro para a Medicina de Família em Portugal. Cadernos Especiais. Lisboa: APMCG; 1990.

9. Associação Portuguesa dos Médicos de Clínica Geral, Direcção Nacional. Declaração da Madeira. Lisboa: APMCG; 1998. Disponível em: URL [http://www.apmcg.pt/files/54/documentos/20070601155323171060.pdf ] Acedido em 8/12/2010

10 Associação Portuguesa dos Médicos de Clínica Geral, Direcção Nacional. Medicina Geral e Familiar. Colapso ou ressurgimento. Lisboa: APMCG; 2003.

11. Ministério da Saúde. Decreto-Lei n. ${ }^{\circ} 117 / 98$ de 5 de Maio de 1998.

12. Ministério da Saúde. Decreto-Lei n. ${ }^{\circ} 157 / 99$ de 10 de Maio de 1999

13. Ministério da Saúde. Despacho Normativo n. ${ }^{\circ}$ 9/2006

14. Missão para os Cuidados de Saúde Primários. Indicadores de Desempenho para as Unidades de Saúde Familiar. Lisboa 2006.

15. Rosenthal MB, Fernandopulle R, Song HSR, Landon B. Paying For Quality: Providers' Incentives For Quality Improvement. Health Affairs, 23, no.2 (2004):127-141.

16. Glass KP, Pieper LE, Berlin MF. Incentive-Based Physician Compensation Models. J Ambulatory Care Manage, 1999, 22(3), 36-46

17. Ministério da Saúde. Portaria n. ${ }^{\circ}$ 301/2008_de 18 de Abril

18. National Health Service. Quality and Outcomes Framework for General Practice. Disponível em: URL: http://www.qof.ic.nhs.uk/Acedido em 30 Dezembro 2010.

19. American Academy of Family Physicians (AAFP). Pay-For-Performance. Disponível em: URL: http://www.aafp.org/online/en/home/policy/policies/p/payforperformance.printerview.html Acedido em 30 Dezembro 2010.

20. Eldridge C, Palmer N. Performance-based payment: some reflections on the discourse, evidence and unanswered questions. Health Policy and Planning 2009;24:160-166

21. Campbell S, Reeves D, Kontopantelis E, Sibbald B, Roland M, D.M. Effects of Pay for Performance on the Quality of Primary Care in England. N Engl J Med 2009;361:368-78

22. Nelson AR. Pay-for-performance programs: Ethical questions and unintended consequences. Current Clinical Practice 2007; 1:16-8

23. Snyder L, Neubauer RL; American College of Physicians Ethics, Professionalism and Human Rights Committee. Pay-for-performance principles that promote patient-centered care: an ethics manifesto. Ann Intern Med. 2007 Dec 4;147(11):792-4. 
24. Gérvas, J., Pérez Fernández, M. Pago por desempeño («pay for performance» [P4P]). AMF 2008; 4(6): 344-352.

25. Rosenthal MB, Frank RG, Li Z, Epstein AM. Early experience with payfor-performance: from concept to practice. JAMA. 2005 Oct 12;294(14):1788-93.

26. Petersen LA, Woodard LD, Urech T, Daw C, Sookanan S. Does Pay-forPerformance Improve the Quality of Health Care? Ann Intern Med. 2006; 145:265-272.

27. Portaria n. ${ }^{\circ} 301 / 2008$ de 18 de Abril. Diário da República, 1. ${ }^{\text {a }}$ série N. ${ }^{\circ} 77-18$ de Abril de 2008

28. British Medical Association. Quality and Outcomes Framework guidance. 2009. Disponível em: URL (documento disponivel em [http://www.nhsemployers.org] Acedido em 8/10/2010

29. Santos I, Ribeiro I. Indicadores de desempenho na consulta. Rev Port Clin Geral 2009;25:228-36

30. Fleetcroft R, Cookson R. Do the incentive payments in the new NHS contract for primary care reflect likely population health gains? J Health Serv Res Policy. 2006;11:27-31.

31. Administração Central do Sistema de Saúde e Missão para os Cuidados de Saúde Primários. Cálculo de Indicadores de desempenho. Critérios a observar na sua implementação. 2009. Disponível em: URL [http://www.mcsp.min-saude.pt/engine.php?id=3176] Acedido em $6 / 2 / 2010$

32. Direção Geral de Saúde. Diagnóstico Tratamento e Controle da Hipertensão Arterial. Circular Normativa. Ministério da Saúde 2004

33. Melo M.A Prevenção Quaternária contra os excessos da Medicina. Rev Port Clin Geral 2007;23:289-93

34. Werner RM, Asch DA. Clinical concerns about clinical performance measurements. Ann Fam Med. 2007;5:159-63.
35. Snyder L, Neubauer RL for the American College of Physicians Ethics, Professionalism and Human Rights Committee. Pay-for-performance principles that promote patient-centered care: an ethics manifesto. Ann Intern Med. 2007; 147:792-4

36. McDonald R, Roland M. Pay for Performance in Primary Care in England and California: Comparison of Unintended Consequences. Ann Fam Med 2009;7:121-127

37. Administração Central do Sistema de Saúde Departamentos de Contratualização dasAdministrações Regionais de Saúde e Missão para os Cuidados de Saúde Primários. Metodologia de Contratualização para 2010. 2010. Disponível em: URL [http://www.mcsp.min-saude.pt/Imgs/ content/page_46/metodologiacontratualizacousf_2010_publicada.pdf] Acedido em 6/4/2010

38. Pinto, D, Corte-Real S, Nunes JM...Actividades preventivas e Indicadores: quanto tempo sobra? Rev Port Clin Geral 2010;26:455-64

39. Starfield B. Family medicine should shape reform, not vice versa. Fam Pract Manag 2009; 16: 6-7.

\section{CONFLITOS DE INTERESSE}

O autor declara não exisitir conflitos de interesse na elaboração deste artigo

\section{ENDEREÇO PARA CORRESPONDÊNCIA}

Jaime Correia de Sousa

Rua Pedro Hispano, $148-6^{\circ}$ Cntr

4100-393 Porto

E-mail: jaimecsousa@gmail.com

Recebido em 29/12/2010

Aceite para publicação em 12/01/2011

\section{ABSTRACT}

\section{PERFORMANCE INDICATORS CONTRACTED WITH FAMILY HEALTH UNITS: A PROGRESS REPORT ONTHE CUR- RENT MOMENT OF PRIMARY HEALTH CARE REFORM IN PORTUGAL}

Performance indicators have been in use in Portugal for some time now for performance assessment and contractualisation in Family Health Units (USFs). It is important to consider both the quality of the performance indicator and its impact on the delivery of health care in general practice in order to improve performance assessment. When performance indicators assess only a limited area of clinical practice or set very high targets, they may limit the focus of clinical activities with possible negative consequences for patients. One of these consequences may be the replacement of Patient-Centred Medicine with Indicator-Based Medicine. In addition, there is poor scientific evidence for the use of some of the indicators and controversial usefulness of others. The difficulty of measuring some health outcomes can produce medicalisation, consumerism, and iatrogenic disease.

In this paper, the authors present some suggestions for improving existing performance indicators. Further developments of related matters, such as the medical information system and contract schemes, are also proposed.

Payment for performance should be based on indicators that produce better health outcomes for patients while respecting the principles of family medicine.

Keywords: Reimbursement; Incentive; Quality Indicators; Health Care; Quality Assurance; Health Care. 